\title{
Les femmes, les coquillages et la mangrove
}

Fisherwomen, shellfish and mangrove

\section{Ariadna Burgos et Peyo Dillais}

\section{(2) OpenEdition}

Journals

Édition électronique

URL : https://journals.openedition.org/tc/6748

DOI : $10.4000 /$ tc. 6748

ISSN : 1952-420X

\section{Éditeur}

Éditions de l'EHESS

\section{Édition imprimée}

Date de publication : 15 décembre 2012

Pagination : 326-337

ISBN : 978-2-7351-1534-

ISSN : 0248-6016

\section{Référence électronique}

Ariadna Burgos et Peyo Dillais, "Les femmes, les coquillages et la mangrove », Techniques \& Culture [En ligne], 59 | 2012, mis en ligne le 23 juillet 2013, consulté le 29 septembre 2022. URL : http:// journals.openedition.org/tc/6748; DOI : https://doi.org/10.4000/tc.6748 


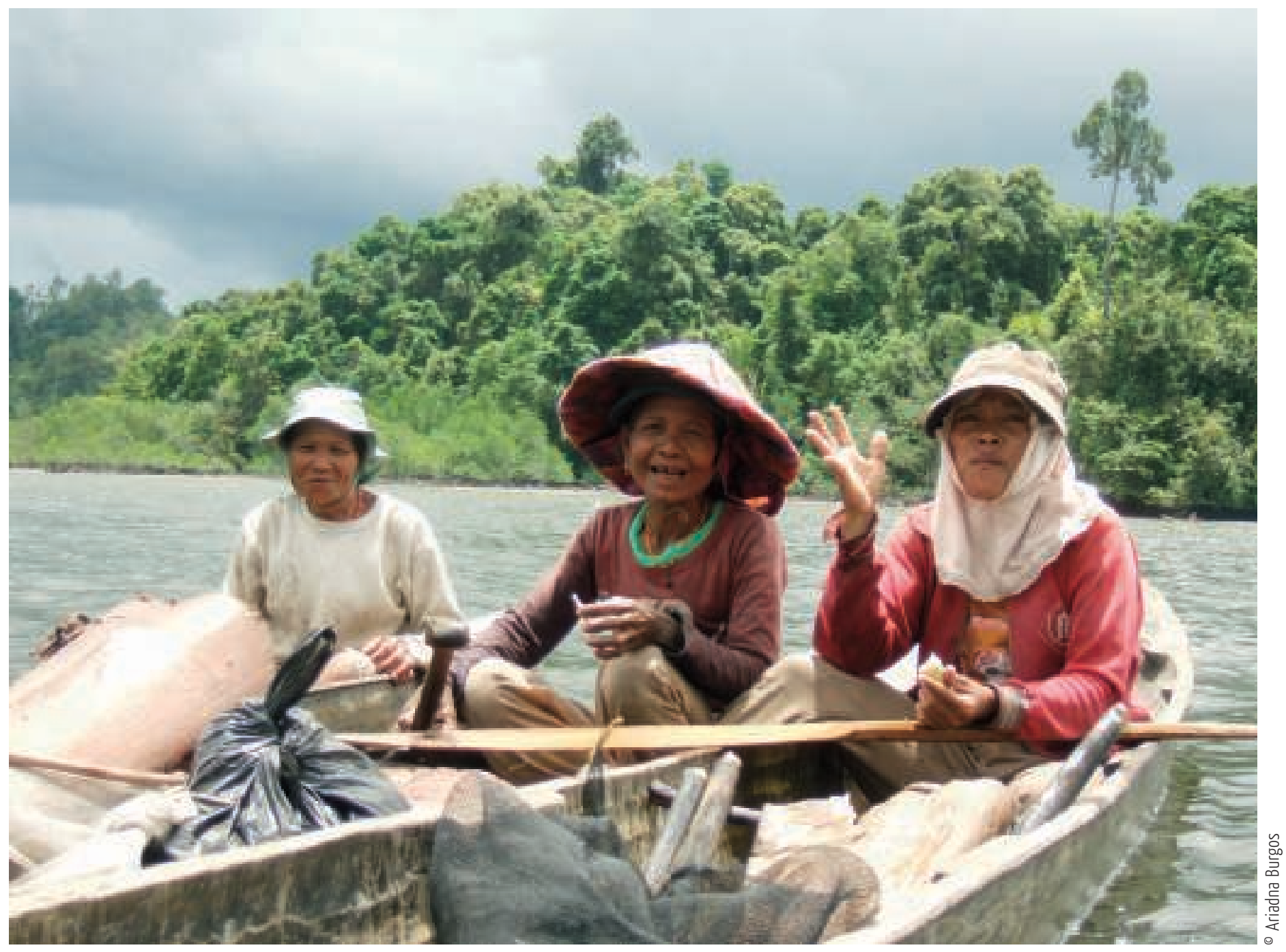




\section{LES FEMMES, LES COQUIILLAGES ET LA MANGROVE}

\section{Collecte d'Anodontia philippiana et Austriella corrugata sur l'île de Siberut (Indonésie)}

Cet article est le fruit des observations et des échanges réalisés auprès des femmes pêcheusescueilleuses de l'île de Sibérut (Indonésie) à l'occasion de deux terrains entre 2010 et 2011. Durant neuf mois, j'ai suivi ces femmes dans la mangrove, ce qui m'a permis de comprendre comment elles parviennent à utiliser leur milieu et à agir sur lui. Elles évaluent, gèrent et utilisent les ressources de leur environnement. Une technique originale a retenu mon attention, celle de l'extraction de deux espèces de bivalves de la famille des Lucinidae, Anodontia philippiana (Reeve 1850) et Austriella corrugata (Deshayes 1843), car elle est difficile à effectuer, réclame de la précision. Les documents qui suivent relatent le travail de ces femmes, leurs savoirs sur les coquillages et décrit, à travers leurs différents mouvements, la technique mise en œuvre pour l'extraction de cette nourriture importante pour elles-mêmes et leurs familles.

Les dépôts et amas de coquillages mis au jour par les archéologues montrent que dès la préhistoire, les mollusques sont consommés dans plusieurs régions du monde (Volman 1978, Waselkov 1987, Faulkner 2009). Limportance des mollusques dans le régime alimentaire de nombreuses sociétés indigènes a été signalée dès le xvIII siècle par d'éminents naturalistes et explorateurs comme Labillardière (1799: 52-54) ou Cook (Beaglehole 1955 : 312) ou même Darwin (Stuart 1977 : 263-266). Dans les trente dernières années on s'est intéressé à leur valeur nutritionnelle et économique (Meehan 1982, Sikorski et Zdzislaw 1990, Carpenter et Niem 1998, Thomas 2003). Cependant les techniques de récolte de coquillages et les savoirs malacologiques n'ont reçu que peu d'attention de la part des ethnologues (Des Rochers 1992, Moss 1993, Thomas 2003) même si cette activité ancienne est toujours d'une importance socio-économique notable pour bon nombre de populations côtières (Primavera et al., 2002, Bonomo 2007, Rondinelli \& Barros 2010). 
C'est le cas des Mentawai, un peuple de pêcheurs-chasseurs-cueilleurs habitant sur l'île de Sibérut ${ }^{1}$, située à environ $130 \mathrm{~km}$ au large des côtes occidentales de Sumatra (Indonésie). La récolte de coquillages y est une activité de subsistance essentielle, qui se pratique dans les nombreux bras d'eau qui divisent les vallées et les plaines de l'île. C'est notamment dans la forêt de palétuviers que cette activité prend une ampleur et une importance considérables.

Dans la Baie de Katuraï, au sud-est de l'île de Sibérut, les femmes partent tous les matins dans la mangrove à la recherche de crabes, de poissons et de coquillages. Elles ont la charge de pourvoir aux besoins quotidiens de toute la famille. Les hommes travaillent davantage à la construction de pirogues et de maisons, à la gestion des champs cultivés, mais aussi à la chasse et l'élevage de sangliers ${ }^{2}$. Dans leur enfance, les garçons accompagnent leur mère dans la mangrove et participent à la collecte de quelques coquillages. Mais une fois adolescents, ils ne s'y aventurent que pour la coupe de bois d'œuvre. Depuis quelques années, certains jeunes hommes partent aussi à la recherche de crabes, seule ressource locale qui soit actuellement commercialisée et exportée vers Padang.

Les femmes, depuis leur enfance, ont une relation étroite avec la mangrove et ont consolidé par leur expérience et la transmission de savoirs, un corpus de connaissances naturalistes qui leur permet d'évaluer leur milieu, d'identifier ses changements et d'optimiser leur travail.

La récolte de coquillages est un travail aussi important que la pêche dans la baie. Selon les femmes, cette activité est « plus fiable », car les coquillages sont relativement sédentaires et "prévisibles ». Par contre, la pêche est une activité dont le rendement est aléatoire et fortuit. Ainsi, les journées de récolte de coquillages garantissent aux femmes une ressource alimentaire à haute valeur protéique pour l'ensemble de la famille (Thomas 2003).

Les journées de travail sont longues et les femmes se déplacent jusqu'à $12 \mathrm{~km}$ en pirogue par jour. Chacune part dans sa pirogue personnelle, mais elles se déplacent souvent en groupe, ou se donnent rendez-vous grâce à un système de toponymes très précis qui leur permet de se retrouver et de se situer dans n'importe quelle partie de la forêt de palétuviers de la baie.

Les savoirs naturalistes en relation avec les différents coquillages sont considérables: les femmes connaissent très bien la diversité et les associations écologiques des différentes espèces, leurs biotopes spécifiques et leur localisation et abondance dans la baie. Elles détiennent aussi un ensemble de savoirs historiques sur les transformations écologiques et sociales du milieu, les pratiques d'exploitation des ressources malacologiques et les événements marquants (naturels ou anthropogéniques) qui ont conduit à la diminution du stock d'une espèce particulière.

L'écosystème de la mangrove comporte un ensemble de micro-habitats et une succession d'assemblages forestiers qui varient selon la salinité, l'influence des marées, la nature et le relief du sol. Cinq grands types d'écosystèmes de mangrove sont dénommés par les Mentawai. Ces « unités écologiques » sont caractérisées par un cortège de bivalves et de gastéropodes occupant différentes niches écologiques. Ainsi, certains bivalves se trouvent enfouis en profondeur dans le sol, d'autres se situent en surface ou sont légèrement enfouis dans la vase, d'autres encore sont fixés sur les racines des palétuviers. En revanche, les gastéropodes se déplacent davantage sur la vase et grimpent sur les troncs: certaines espèces se positionnent sur le bois en décomposition tandis que d'autres préfèrent monter sur les feuilles des palétuviers. 
Au total, il existe 45 espèces de bivalves et de gastéropodes, dont 38 sont très appréciées dans l'alimentation. Cette diversité de mollusques est aussi remarquable que la diversité des techniques employées pour les extraire. Les femmes possèdent ainsi des techniques de récolte qui varient et qui sont conditionnées par l'habitat de l'espèce recherchée et par la hauteur de la marée. La plupart de ces techniques sont des techniques du corps, au sens maussien où le corps « est l'instrument naturel et principal » (Mauss 1929), qui permet, à partir de mouvements variés et coordonnés, l'identification et la récolte des coquillages.

Une des techniques d'extraction les plus employées à Katuraï est le pasigutgut. Cette technique traditionnelle et unique, est utilisée pour déterrer le taïknuktuk (Anondontia philippiana) et le sikoïra (Austriella corrugata), deux bivalves qui se situent à proximité des racines de Rhizophora apiculata et $R$. mucronata, enfouies à environ $80 \mathrm{~cm}$ de profondeur dans la vase. La technique dite "pasigutgut » s'effectue en général avec $70 \%$ du corps immergé dans l'eau et de préférence lors de la marée basse. Les femmes se placent à proximité des racines, et s'en servent comme appui pour se stabiliser sur la vase. Le bas du corps plongé dans l'eau, elles exercent avec leurs pieds un mouvement de pression répétitif et vertical pour pénétrer en profondeur dans la vase. Avec leurs doigts de pieds, elles explorent le substrat et arrivent aisément à différencier les bouts de bois des coquillages. Malgré la ressemblance physique entre les coquilles du taiknuktuk et du sikoira (cf. p333), les femmes de la mangrove font preuve d'une sensibilité et d'un sens du toucher très développés: elles savent parfaitement différencier les deux espèces en utilisant uniquement leurs orteils.

La quête des coquillages implique un effort physique important: les femmes explorent tous les recoins de la mangrove pieds nus, grimpent sur les racines, soulèvent les morceaux de bois mort, marchent à moitié pliées, plongent dans les rives de la baie et à l'intérieur des cours d'eau. Elles sont couvertes de vase, d'eau et de sel durant toute la journée. C'est un travail très pénible où elles sont également exposées à de nombreux types de blessures à cause des échardes, des morceaux de bois pointus, des bords effilés des huîtres fixées aux palétuviers et des piqûres incessantes de moustiques.

Lorsque les femmes se retrouvent, elles se questionnent sur le rendement de la journée de l'une et de l'autre et sur son site de travail. Elles se renseignent également sur la position et les activités des autres femmes, elles commentent les actualités des villages de la baie et échangent leurs prévisions climatiques du jour et de la semaine. Elles examinent sans vergogne leurs récoltes respectives, en soulevant les paniers et en regardant dans le fond de la pirogue les différents produits collectés. Cela peut donner lieu à quelques échanges, celles ayant pêché davantage faisant don, ou prêtant une petite partie de leur «butin » aux autres.

Selon les femmes, la collecte de coquillages est une activité possible toute l'année: il n'y a pas une saison particulière dans laquelle les différentes espèces de coquillages sont plus abondantes. Ainsi, le choix de l'activité et l'articulation du calendrier de collecte et d'extraction de coquillages ne reposent pas exclusivement sur la disponibilité et l'accessibilité aux mollusques, mais sur la réévaluation continuelle de la disponibilité de toutes les autres ressources alimentaires.

Les profondes transformations socio-économiques et culturelles qui ont eu lieu durant les cinquante dernières années dans la baie de Katuraï, et à Sibérut en général (Persoon 2003, Forestier et al. 2008 : 144), mettent en péril l'exploitation durable des ressources de la mangrove à long terme. De nos jours, les besoins financiers créés par de nouvelles obligations telles que la scolarisation des enfants, l'essence et les vêtements, se font de 
plus en plus pressants dans la baie. La collecte de coquillages y est toujours une activité de subsistance durable et traditionnelle. Mais les ressources malacologiques reposent sur un équilibre fragile qui serait menacé si les opportunités et la demande du marché venaient à évoluer rapidement: notamment par le développement de voies d'acheminement et de conditionnement des stocks.

C'est d'ailleurs ce qui s'est produit pour un bivalve, le bebeleh (Anadara granosa). Selon les doyennes des pêcheuses-collectrices, ce grand coquillage se trouvait autrefois en abondance sur les bancs de vase des mangroves littorales ${ }^{3}$. Suite à une forte demande nationale et internationale, elle a été la cible d'une exploitation intensive dans les années quatre-vingt-dix et exportée en masse vers Padang: c'était à l'époque l'une des seules opportunités d'accès à l'argent, et les villageois n'ont pas hésité à en profiter et aujourd'hui, le bebeleh est une espèce extrêmement rare dans la baie de Katurai. Malgré les incertitudes qui pèsent sur la durabilité des ressources de la mangrove, « il y a de la nourriture si on va la chercher » affirment toujours les femmes. Et au fil des innombrables bras d'eau de cette forêt de palétuviers résonnent les chants, les éclats de rires et de voix, des pêcheuses qui s'interpellent à distance pour savoir si tout se passe bien.

\section{NOTES}

En ouverture: Les femmes de la mangrove (île de Siberut) dans leurs pirogues lors d'une halte sur le chemin de la collecte des coquillages ( $\mathrm{Cl}$. A. Burgos).

1. Les Mentawai sont les habitants de l'archipel de Mentawai, un archipel composé de quatre îles. L'île de Sibérut est la plus septentrionale et la plus vaste de l'archipel de Mentawai, avec $4000 \mathrm{~km}^{2}$.

2. La chasse et l'élevage de sangliers sont plus rares dans cette zone de l'île qu'à l'intérieur où la chasse est toujours une activité largement répandue. À Katuraï, la chasse se pratique moins fréquemment, et la chair de sanglier n'est finalement consommée que lors des cérémonies, rituels et événements occasionnels par l'ensemble du clan.
3. Anadara granosa (Linnaeus, 1758) est une espèce à haute valeur économique dans toute l'Asie du Sud-Est (Faulkner 2009). Elle se caractérise par une coquille épaisse et très résistante, et par sa capacité de conservation de l'ordre de vingt jours après sa récolte, ce qui facilite son transport et son stockage. 


\section{RÉFÉRENCES}

Bonomo, M. 2007 El uso de los moluscos marinos por los cazadores-recolectores pampeanos. Chuungara 39 (1) : 87-102.

Beaglehole, J.-C. (dir.) 1955 Journals of Captain James Cook on his voyage discovery. Vol 1: The Voyage of the Endeavor, 1768-1771. Cambridge : Cambridge University press.

Carpenter, K. E., Niem, V. H. (dir.) 1998 FAO Species Identification Guide for Fishery Purposes. The Living Marine Resources of the Western Central Pacific. Seaweed, Corals, Bivalves and Gastropods. Rome : FAO, Vol 1. 686 p.

Des Rochers, K. 1992 Women's Fishing on Kosrae: a Description of Past and Present Methods. Micronesica 25(1):1-22.

Faulkner, P., 2009 Focused, Intense and Long-Term: Evidence for Granular Ark (Anadara granosa) Exploitation from Late Holocene Shell Mounds of Blue Mud Bay, Northern Australia. In Journal of Archeological Science 36(3): 821-834.

Forestier, H., Guillaud, Meyers, K., Simanjuntak, T. 2008 Mentawai : L'̂̂le des hommes fleurs. Sommières : IRD - Romain Pages, 157 p.

Labillardière, J. 1799 Relation du voyage à la recherche de La Pérouse. Paris : Jansen G.H.J. Vol 2. 332 p.

Mauss, M., 1929 Intervention à la suite d'un exposé de MM. Guillaume et Meyerson à l'Institut français d'anthropologie [Débat sur l'origine de la technologie humaine], Anthropologie 39, p. 130 (CEuvres, t. III, 1969, Paris : Éditions de Minuit, p. 257).

Meehan, B., 1982 Shell Bed to Shell Midden. Canberra : Australian Institute of Aboriginal Studies, 189 p.

Moss, M., 1993 Shellfish, Gender, and Status on the Northwest Coast: Reconciling Archeological, Ethnographic, and Ethnohistorical Records of the Tlingi. American Anthropologist 95 (3): 631-652.

Persoon, G. 2003 Conflicts Over Trees and Waves on Siberut Island. Geografiska Annaler 85B (4) : 253-264.

Primavera, J.H, Lebata, M.J., Gustilo, L.F. \& J.P. Altamirano 2002 Collection of the Clam Anodontia edentula in Mangrove Habitats in Panay and Guimaras, central Philippines. Wetlands Ecology and Management 10: 363-370.

Rondinelli, S.F., Barros, F. 2010 Evaluating Shellfish Gathering (Lucina pectinata) in a Tropical Mangrove System. Journal of Sea Research 64 (3): 401-407.

Sikorski, Zdzislaw, E. 1990 Seafood: Resources, Nutritional Composition, and Preservation. Boca Raton, Fla : CRC Press, $256 \mathrm{p}$.

Stuart, D. E. 1977 Seasonal Phases in Ona Subsistence, Territorial Distribution and Organization. In Lewis R. Binford (dir.) For Theory Building in Archaeology: Essays on Faunal Remains, Aquatic Resources, and Systemic Modeling. New York: Academic Press, 419 p.

Thomas, F.R. 2003 Shellfish Gathering in Kiribati, Micronesia: Nutritional, microbiological, and toxicological aspects. Ecology of Food and Nutrition 42 (2): 91-127.

Volman, T. P. 1978 Early Archeological Evidence of Shellfish Gathering. Science 201 : 911-913.

Waselkov, G. A. 1987 Shellfish Gathering and Shell Midden Archaeology. In M. B. Schiffer Advances in Archaeological Method and Theory 10. New York : Academic Press : 93-210.

\section{MOTS-CLÉS}

Mangrove, coquillages, femmes, techniques de collecte, savoirs locaux, ethnosciences

\section{KEYWORDS}

Mangrove, shellfish, fisherwomen, collecting techniques, local knowledge, ethnoscience 
Les femmes de Katurä explorent toutes les criques et les ilots de la mangrove à la recherche du sik oira (Austriella corrugata) et du taik nuk tuk (Anodontia philippiana).

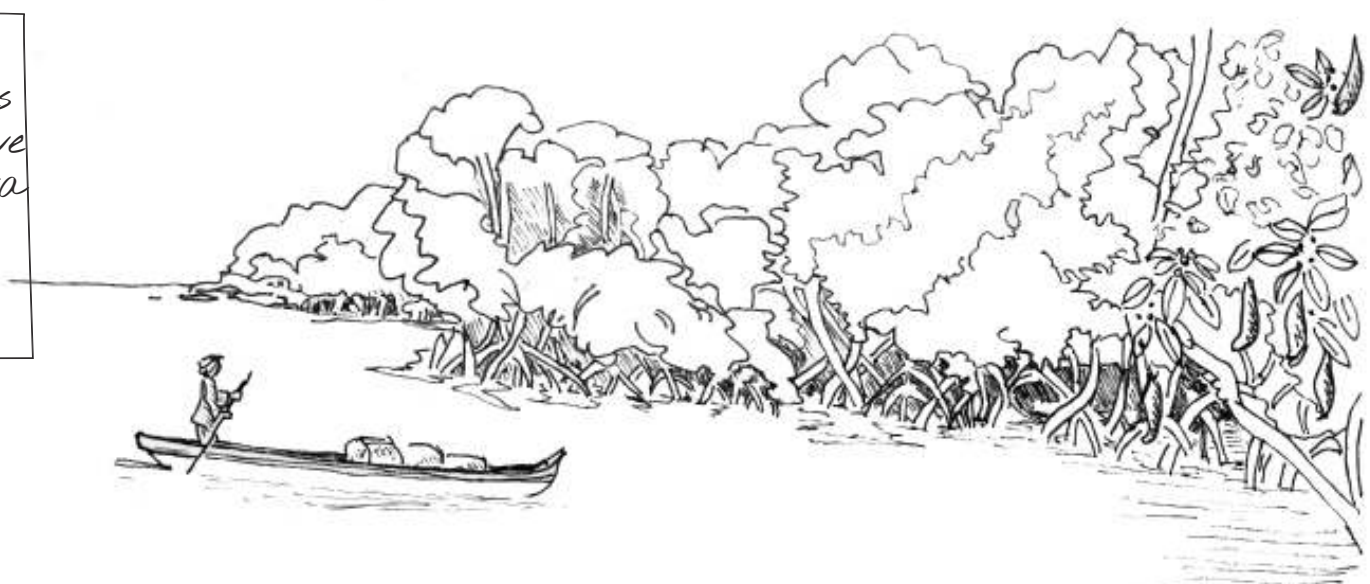

Les femmes partent en groupe ou se donnent rendezvous dans la mangrave. Grâce à un système de référence geogr aphique trés developpe comportant à la fois des toponymes et un vocabulaire géomorphologique très précis, elles parviennent à se retrouver dans nimporte quel site de la baie.

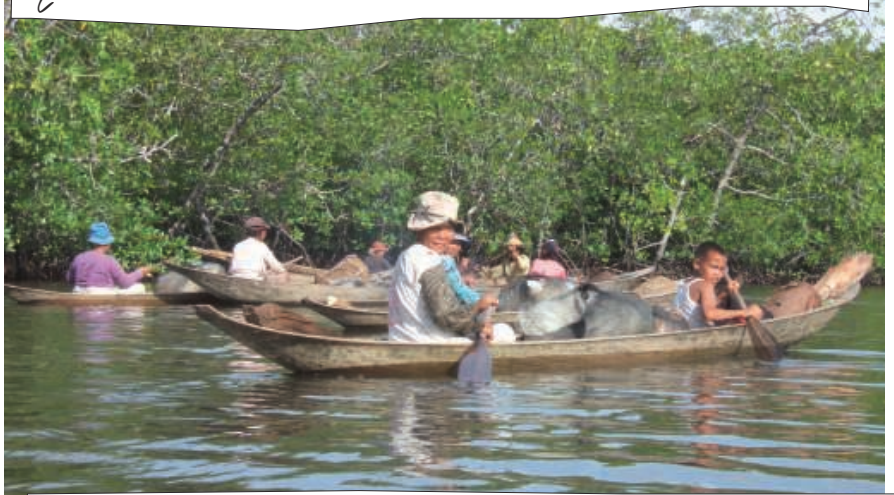

La collecte de taïknuktuk et sikoira est reservee aux femmes et aux filles addescentes. Les enfants ne participent pas à cette pêche, elle est consideree comme une activite à risque qui requiert de l'experience.

Ces deux bivalves sont enfouis à $80 \mathrm{~cm}$ dans la vase à proximite des racines de Rhizophora apiculata et $R$ mucronata. Pour les deterrer, les femmes se tiennent fermement aux racines des palétuviers le bas du corps plonge dans lieau, les jambes dans la vase, elles cherchent avec leurs pieds les coquillages.

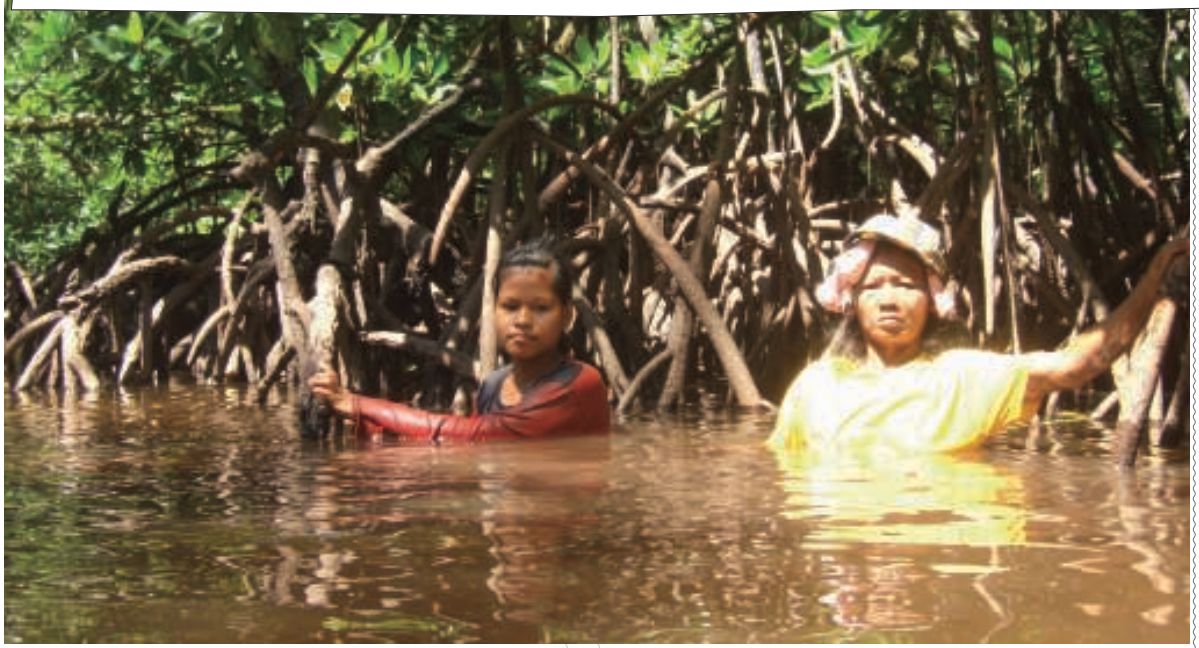

Selon les femmes, Lorsque le sol est très mou, on trouve plus de taïknuktuk, akors que dans un sol légerement plus ferme, on trouve plutôt des sikoira. 


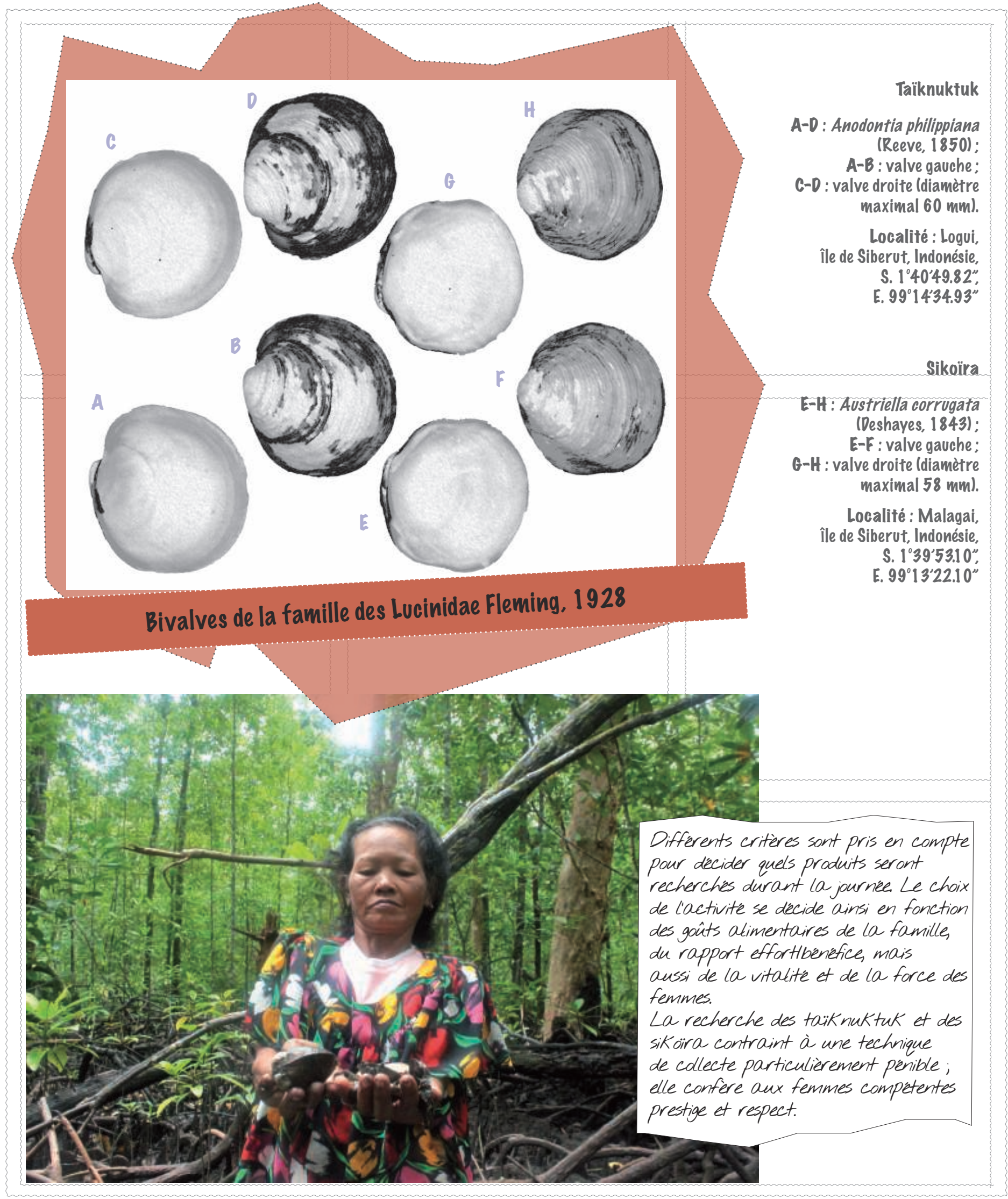




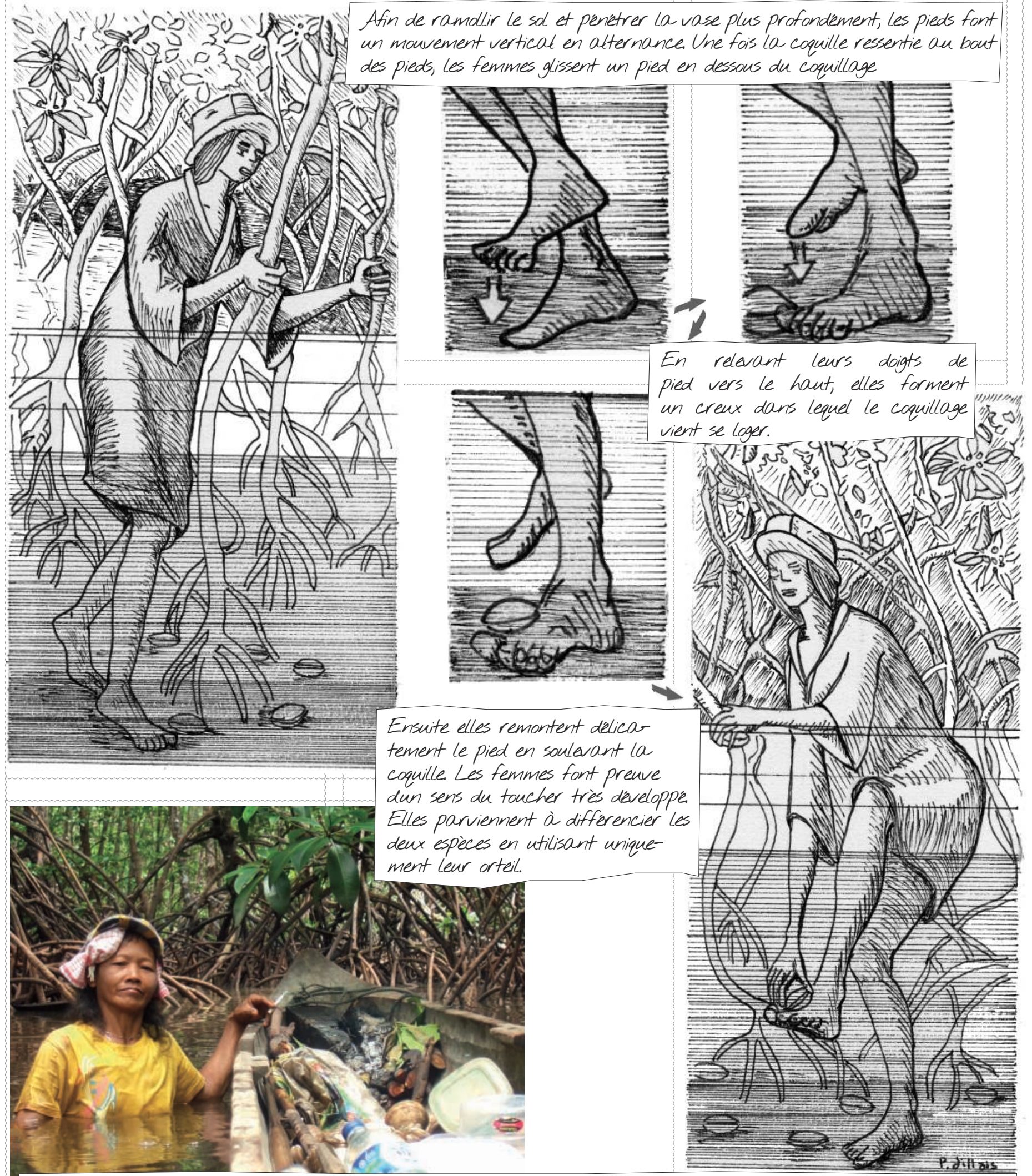

Dans leur pirogue, les femmes emportent le strict nécessaire de leau, du sagu (une farine extraite dun bois de palmier et cuite dans du bambou), le bubuket (une büche qui se consume lentement dur ant la journee, fait office de briquet et dont la fumee éloigne les moustiques), une machette, un panier en osier, et des cigarettes. Les femmes travaillent durement, et la pause cigarette est leur seul moment de détente. 
A la mi-journée, les femmes confectionnent leur coin cuisine, le purrusuat, à l'arrière de la pirogue où elles cuisent quelques coquillages et les mangent. Le purrusuat est constitue de trois couches: dabord quelques racines de Rhizophora, puis trois poignees de vase, et enfin des fewilles de Rhizophora. La femme pose une büche, le bubuket, qui se consume lentement.
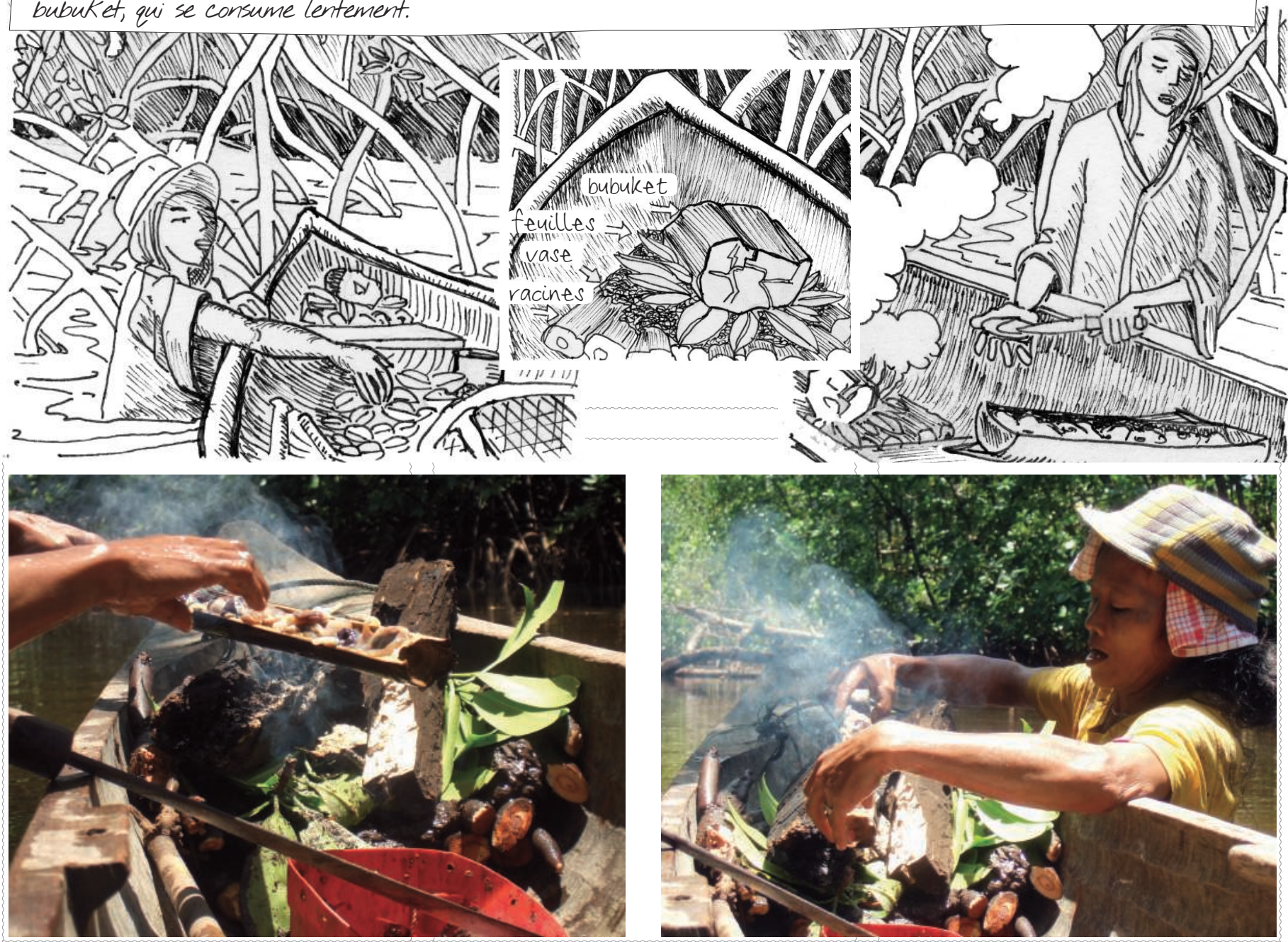

Selon les femmes, les plus grands risques lors de la quête de ta"k nuk tuk et de sik oira sont les coupures aux pieds, les infections à linterieur des ongles et entre les doigts de pieds, et les serpents au venin mortel qui évoluent dans la vase.

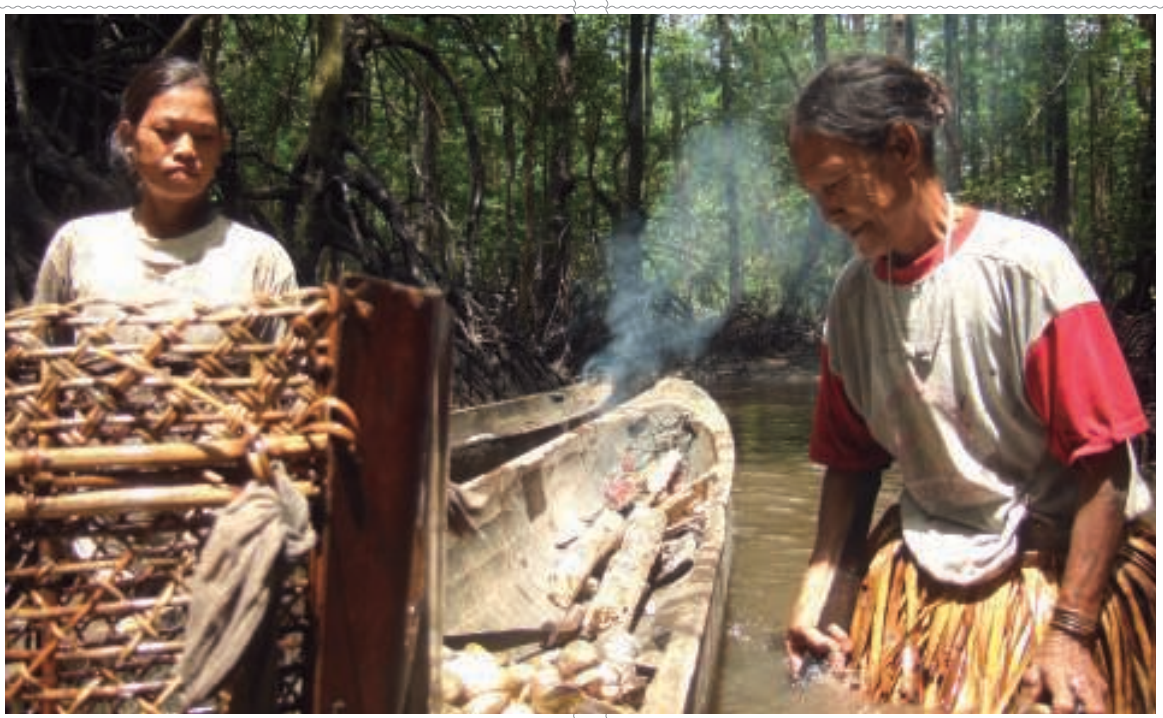



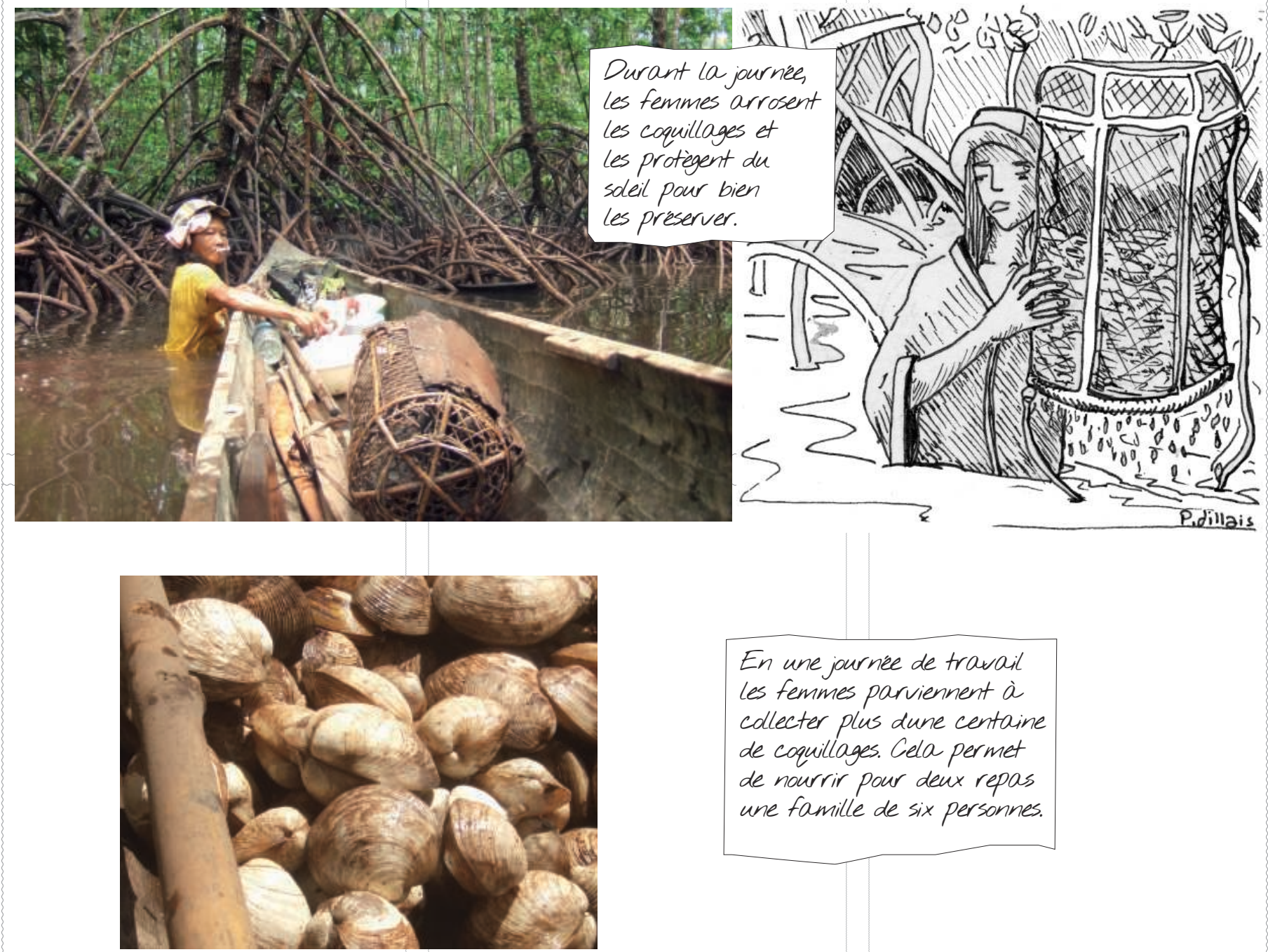

En une journee de travail les femmes parviennent à collecter plus dune centaine de coquillages. Cela permet de nourrir pour deux repas une famille de six personnes.

\section{1) QAN EAll}

Une fois leur panier bien rempli, après environ 8 heures de travail, les femmes rentrent au village.

Les enfants attendent leur miere à l'embarcadere, impatients de savoir ce quelle leur apporte a manger à son retour de la mangrave.

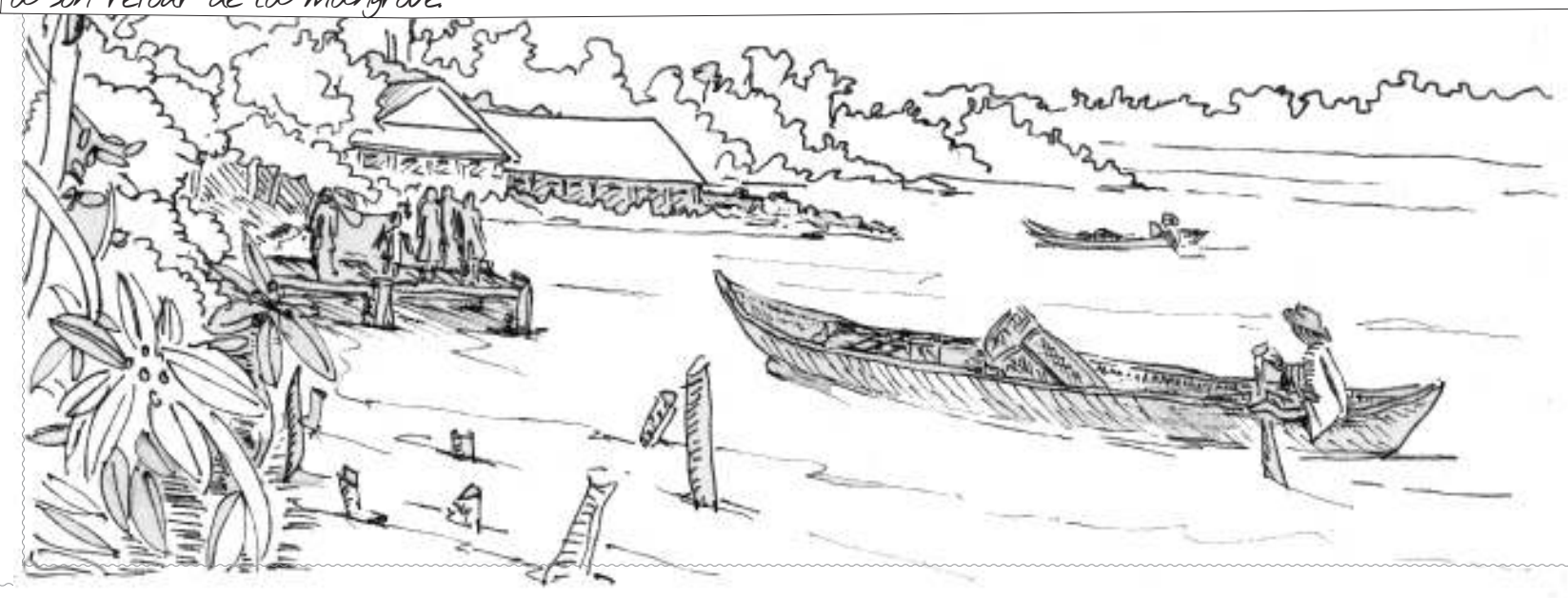




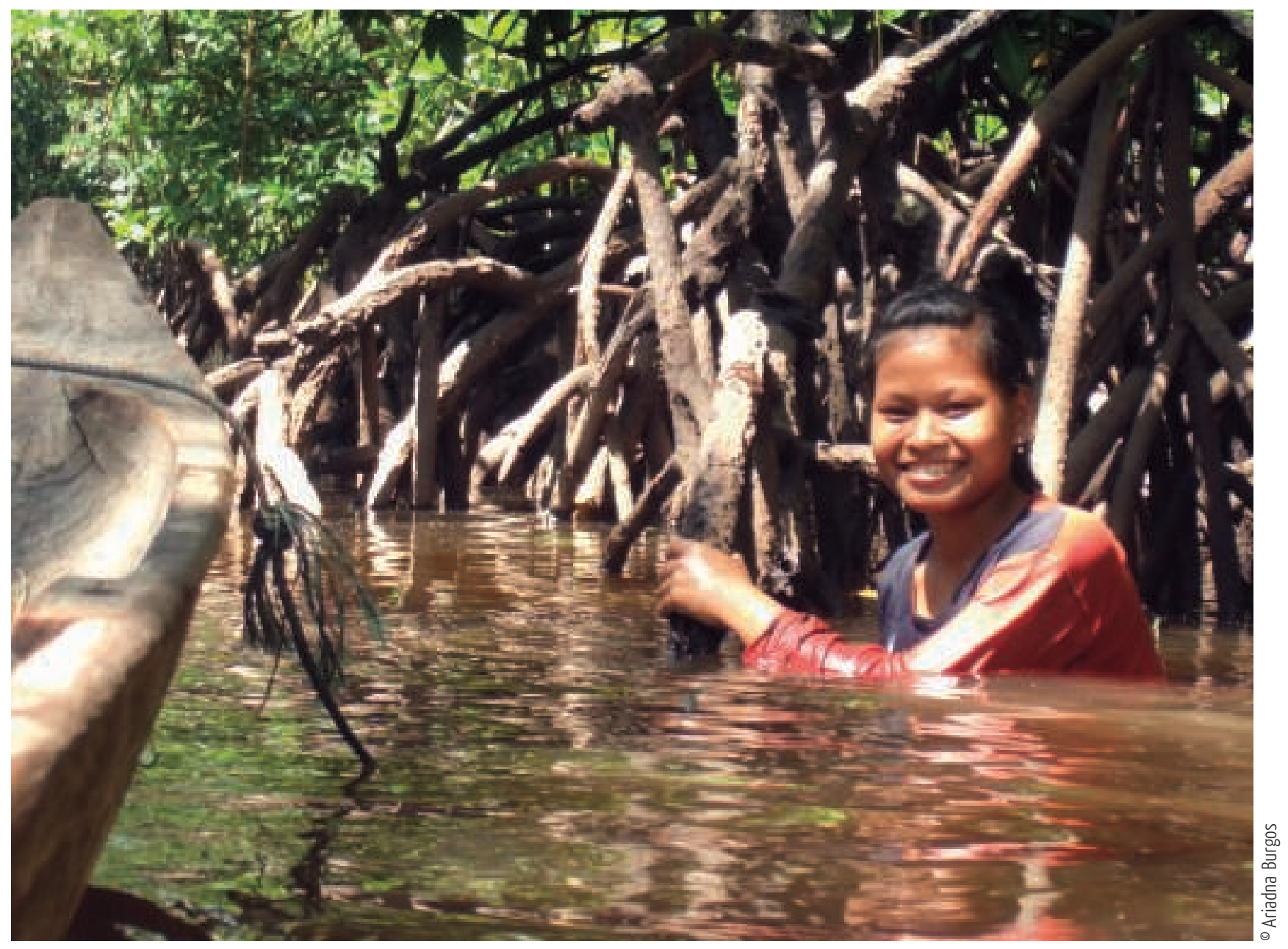

